\title{
Optimal Time-Consistent Investment Strategy for a DC Pension Plan with the Return of Premiums Clauses and Annuity Contracts
}

\author{
De-Lei Sheng and Ximin Rong \\ Department of Mathematics, Tianjin University, Tianjin 300072, China \\ Correspondence should be addressed to De-Lei Sheng; tjhbsdl@126.com
}

Received 3 March 2014; Accepted 1 June 2014; Published 24 June 2014

Academic Editor: Xiang Li

Copyright (C) 2014 D.-L. Sheng and X. Rong. This is an open access article distributed under the Creative Commons Attribution License, which permits unrestricted use, distribution, and reproduction in any medium, provided the original work is properly cited.

Defined contribution and annuity contract are merged into one pension plan to study both accumulation phase and distribution phase, which results in such effects that both phases before and after retirement being "defined". Under the Heston's stochastic volatility model, this paper focuses on mean-variance insurers with the return of premiums clauses to study the optimal timeconsistent investment strategy for the DC pension merged with an annuity contract. Both accumulation phase before retirement and distribution phase after retirement are studied. In the time-consistent framework, the extended Hamilton-Jacobi-Bellman equations associated with the optimization problem are established. Applying stochastic optimal control technique, the time-consistent explicit solutions of the optimal strategies and the efficient frontiers are obtained. In addition, numerical analysis illustrates our results and also deepens our knowledge or understanding of the research results.

\section{Introduction}

Annuity contract and defined contribution are merged into one pension plan to study both accumulation phase and distribution phase, which results in such effects that both phases before and after retirement being "defined," making the defined contribution plans even more portable and of great convenience for insurance companies.

Annuity is any financial contract providing continuing payment with a fixed total amount on fixed time interval which usually can be once a year. A lot of research have been done on annuity plans and have gained many good results. For example, Gao [1] investigated annuity contracts in the optimal investment problem under the constant elasticity of variance model in 2009.

Defined contribution (DC) pension plan is a type of retirement plan in which fixed contributions are paid into an individual account, and then the contributions are invested in a financial market and the returns on the investment (positive or negative) are credited to the individual's account. Only contributions to the account are guaranteed, but the future benefits fluctuate on the basis of investment earnings (referring to Cairns et al. [2], etc.). A pension member contributes a predetermined amount of money as premiums before retirement, which lasts for the whole working period of the pension member. From the moment the member retires, the accumulation phase end and the fund will be distributed monthly as old-age pension. Obviously, the distribution per month is not predetermined; however, it is determined by the whole accumulation fund size.

Different from the defined contribution (DC) pension plans, a defined benefit plan is "defined" in the sense that the benefit formula is defined and known in advance, which is based on the earnings history, tenure of service, and age, rather than depending on individual investment returns directly. Because of the cost of administration being fewer than defined benefit plans and ease of determining the plan sponsor's liability in practice, defined contribution plans have been widespread all over the world as the dominant form of plan in many countries.

For the reason that the retirement benefits of the DC pension plan depend on the fund size, the insurer must invest 
on financial markets to increase the returns, which results in the optimal investment problem becoming so crucial that lots of interests attracted into this field. Dokuchaev and Yu Zhou [3] studied optimal investment strategies with bounded risks, general utilities, and goal achieving. Blanchet-Scalliet et al. [4] investigated optimal investment decisions when time horizon is uncertain. Faggian and Gozzi [5] developed dynamic programming approach for a family of optimal investment models with vintage capital. Blake et al. [6] study the optimal asset allocation problem for DC pension funds.

In the financial market, the price process of stock is described by Heston's stochastic volatility (SV) model. Heston's stochastic volatility (SV) is a classical stochastic volatility model. In the previous literatures, Heston's SV model was very popular for option pricing; however, there are few literatures about the investment problem for insurers. Kraft [7] began to apply the Heston model to study the portfolio problem By maximizing utility from terminal wealth with respect to a power utility function. Li et al. [8] apply the Heston's SV model to investigate the reinsurance and investment problem under the mean-variance criterion.

Mean-variance criterion is first proposed by Markowitz [9] to investigate portfolio selection. But the optimal strategies under the mean-variance criterion are not time consistent, because the mean-variance criterion lacks the iterated expectation property so that the Bellman's principle of optimality does not hold. However, in many situations time consistency of strategies is a basic requirement for rational decision makers. Recently, many researchers paid much attention to time-inconsistent stochastic control problems and aimed at deriving the optimal time-consistent strategies. In 2010, Bjork and Murgoci [10] studied the general theory of Markovian time inconsistent stochastic control problems. Bjork et al. [11] investigated the portfolio optimization with state-dependent risk aversion in the mean-variance framework in 2012.

Some pension plan members may die early during the accumulation phase so that they have no chance to accept pension distribution after retirement. The DC pension plans must have return of premium clauses to protect the rights of them. With this kind of actuarial clause, the dead member can withdraw the premiums she/he contributes or the premiums accumulated by a predetermined interest rate. For this problem, He and Liang [12] studied the DC pension plan for a mean-variance insurer with the return of premiums clauses in 2013. But they focused on the accumulation phase before retirement while there is no serious research on the other phase after retirement.

As far as we know, there is no literature to study both annuity contract and the DC plan with the return of premiums clauses under Heston's SV models under the meanvariance criterion. In this paper, we study a whole pension plan that the DC pension plan with the return of premiums clauses is merged with annuity contract under the meanvariance criterion to find an optimal time consistent strategy under the Heston's stochastic volatility model which can describe the volatility of risk asset more perfectly. Both accumulation phase before retirement and distribution phase after retirement of pension plan are studied in detail.
This paper proceeds as follows. In Section 2, we formulate the model and introduce the actuarial methods of the DC pension plan with the return of premiums clauses. Section 3 solves the time inconsistent problem in the framework of mean-variance criterion. In Section 4, we give some numerical analysis to demonstrate the results. Section 5 concludes the paper.

\section{Formulation of the Model}

In this paper, the defined contribution in the accumulation phase before retirement is merged with an annuity in the distribution phase after retirement to make one whole pension plan. The contributions are invested in a financial market, which consists of one risk-free asset and a stock, to increase revenues. We try to find the optimal time-consistent investment policy of the DC pension fund for a meanvariance insurer with the return of premiums clauses during the accumulation phase and the benefits of pension fund paid by the form of annuities in distribution phase.

2.1. The Financial Market. Throughout this paper, $(\Omega, \mathscr{F}, P$, $\left.\left\{\mathscr{F}_{t}\right\}_{0 \leq t \leq T}\right)$ denotes a complete probability space satisfying the usual condition, where $T>0$ is a finite constant representing the investment time horizon; $\mathscr{F}_{t}$ stands for the information available until time $t$.

The price $S_{0}(t)$ of bonds is given by

$$
\mathrm{d} S^{0}(t)=r S^{0}(t) \mathrm{d} t, \quad S^{0}(0)=1 .
$$

The price $S(t)$ of equities obeys the Heston's stochastic volatility model:

$$
\begin{array}{r}
\mathrm{d} S(t)=S(t)\left[(r+\lambda L(t)) \mathrm{d} t+\sqrt{L(t)} \mathrm{d} W_{1}(t)\right], \\
S(0)=s_{0}, \\
\mathrm{~d} L(t)=k(\theta-L(t)) \mathrm{d} t+\sigma \sqrt{L(t)} \mathrm{d} W_{2}(t), \\
L(0)=l_{0},
\end{array}
$$

where $r>0$ is the risk-free interest rate and $\lambda, k, \theta, \sigma$ are positive constants and the two Brownian motions satisfying $\mathrm{E}\left[W_{1}(t), W_{2}(t)\right]=\rho \cdot t, \rho \in[-1,1]$ are the correlation coefficients of $W_{1}(t), W_{2}(t)$.

2.2. The Accumulation Phase before Retirement. During the period before retirement, the contributions are invested in a risk-free asset and a stock to maximize the pension fund size at retirement. Let $X(t)$ denote the pension wealth at time $t \in[0, T]$, inspired by He and Liang [12], and we formulate the model of DC pension fund with the return of premiums clauses for Heston's stochastic volatility model as follows.

For the convenience of expression, Let us do some symbol descriptions first.

$P$ denotes the premium per unit time, which is a predetermined variable;

$\omega_{0}$ denotes the accumulation period starting age; 
$T$ is the time length; that is, $\omega_{0}+T$ is the end age of the pension fund accumulation period;

$\delta_{(1 / n), \omega_{0}+t}$ denotes the mortality rate from time $t$ to time $t+(1 / n)$;

$t P$ is the accumulated premium at time $t$;

$t P \delta_{(1 / n), \omega_{0}+t}$ is the premium returned to the dead member from time $t$ to time $t+(1 / n)$.

The plan members who die early can withdraw the premiums she/he contributes or the premiums accumulated at a predetermined interest rate, which is the actuarial return of premiums clause.

To guarantee the interests of pension members, the pension management must invest in equities and bonds to increase the size of pension fund during the accumulation phase.

$\pi$ is the proportion allocated in the equities, which is the control variable;

$1-\pi$ is the remaining allocated in bonds.

First, we formulate the fund size $X(t)$ as a differential form. Taking the time interval by $[t, t+(1 / n)]$,

$$
\begin{aligned}
X\left(t+\frac{1}{n}\right) & \\
= & X(t)\left(\pi \frac{S_{t+(1 / n)}}{S_{t}}+(1-\pi) \frac{S_{t+(1 / n)}^{0}}{S_{t}^{0}}\right) \\
& +P \frac{1}{n}-P t \delta_{(1 / n), \omega_{0}+t} \\
= & X(t)\left(1+\pi \frac{S_{t+(1 / n)}-S_{t}}{S_{t}}+(1-\pi) \frac{S_{t+(1 / n)}^{0}-S_{t}^{0}}{S_{t}^{0}}\right) \\
& +P \frac{1}{n}-P t \delta_{(1 / n), \omega_{0}+t} \\
= & X(t)\left(1+\Delta_{t,(1 / n)}\right)+P \frac{1}{n}-P t \delta_{(1 / n), \omega_{0}+t},
\end{aligned}
$$

where $\Delta_{t,(1 / n)}=\pi\left(\left(S_{t+(1 / n)}-S_{t}\right) / S_{t}\right)+(1-\pi)\left(\left(S_{t+(1 / n)}^{0}-S_{t}^{0}\right) / S_{t}^{0}\right)$.

Using the actuarial formulas to simplify (3), the force function of mortality denoted by $\mu(t)$ and the conditional death probability satisfies

$$
\delta_{t, y}=1-p_{t, y}=1-e^{-\int_{0}^{t} \mu(y+s) \mathrm{d} s} .
$$

So

$$
\delta_{(1 / n), \omega_{0}+t}=1-e^{-\int_{0}^{(1 / n)} \mu\left(\omega_{0}+t+s\right) \mathrm{d} s}=\mu\left(\omega_{0}+t\right) \frac{1}{n}+o\left(\frac{1}{n}\right),
$$

as $n \rightarrow \infty$, and $\mu\left(\omega_{0}+t\right)$ is small during the accumulation phase of the pension plan. Thus

$$
\begin{aligned}
X\left(t+\frac{1}{n}\right)= & X(t)\left(1+\Delta_{t,(1 / n)}\right)+P \frac{1}{n}-P t \delta_{(1 / n), \omega_{0}+t} \\
= & X(t)\left(1+\Delta_{t,(1 / n)}\right)+P \frac{1}{n}-P t \mu\left(\omega_{0}+t\right) \frac{1}{n} \\
& +o\left(\frac{1}{n}\right), \\
X\left(t+\frac{1}{n}\right)-X(t)= & X(t) \Delta_{t,(1 / n)}+P \frac{1}{n} \\
& -\operatorname{Pt\mu }\left(\omega_{0}+t\right) \frac{1}{n}+o\left(\frac{1}{n}\right) .
\end{aligned}
$$

Let $n \rightarrow \infty$, and then

$$
\begin{gathered}
\frac{S_{t+(1 / n)}-S_{t}}{S_{t}} \longrightarrow \frac{\mathrm{d} S_{t}}{S_{t}}, \\
\frac{S_{t+(1 / n)}^{0}-S_{t}^{0}}{S_{t}^{0}} \longrightarrow \frac{\mathrm{d} S_{t}^{0}}{S_{t}^{0}}, \\
\Delta_{t,(1 / n)}=\pi \frac{\mathrm{d} S_{t}}{S_{t}}+(1-\pi) \frac{\mathrm{d} S_{t}^{0}}{S_{t}^{0}} .
\end{gathered}
$$

And (7) becomes

$$
\begin{aligned}
\mathrm{d} X(t)= & X(t)\left[\pi \frac{\mathrm{d} S_{t}}{S_{t}}+(1-\pi) \frac{\mathrm{d} S_{t}^{0}}{S_{t}^{0}}\right] \\
& +P \mathrm{~d} t-P t \mu\left(\omega_{0}+t\right) \mathrm{d} t .
\end{aligned}
$$

Plugging (1) and (2) into (9)

$\mathrm{d} X(t)$

$$
\begin{aligned}
= & X(t)\left[\pi\left((r+\lambda L(t)) \mathrm{d} t+\sqrt{L(t)} \mathrm{d} W_{1}(t)\right)+(1-\pi) r \mathrm{~d} t\right] \\
& +P \mathrm{~d} t-P t \mu\left(\omega_{0}+t\right) \mathrm{d} t \\
= & {\left[X(t)(r+\pi \lambda L(t))+P\left(1-t \mu\left(\omega_{0}+t\right)\right)\right] \mathrm{d} t } \\
& +X(t) \pi \sqrt{L(t)} \mathrm{d} W_{1}(t) .
\end{aligned}
$$

If we choose the mortality force function as the following form:

$$
\mu(t)=\frac{1}{\omega-t}, \quad 0 \leq t<\omega,
$$

where $\omega$ is the maximal age of the life table. Then the SDE (10) becomes

$$
\begin{aligned}
\mathrm{d} X(t)= & {\left[X(t)(r+\pi \lambda L(t))+P \frac{\omega-\omega_{0}-2 t}{\omega-\omega_{0}-t}\right] \mathrm{d} t } \\
& +X(t) \pi \sqrt{L(t)} \mathrm{d} W_{1}(t), \quad 0 \leq t \leq T .
\end{aligned}
$$


The pension management's optimization problem could be described as follows:

$$
\sup _{\pi \in \Pi}\left\{\mathrm{E}_{t, x, l} X^{\pi}(T)-\operatorname{Var}_{t, x, l} X^{\pi}(T)\right\}
$$

where $\prod=\{\pi \mid \pi \in[0, \infty)\}$, which means that a short sell of the bonds is permitted.

2.3. The Distribution Phase after Retirement. Inspired by Gao [1], the whole accumulation fund will purchase a paid-up annuity at retirement time $t=T$ and the purchase rate of annuity will calculate on a predetermined interest rate. The part of the fund used to purchase an annuity of $N$ periods is denoted as $D$, where $D \leq X(T)$. The surplus at the end of the fixed period can be used again in a similar way or paid back to the participants. The contributions benefit to pay between $T$ and $T+N$ are given by

$$
\zeta=\frac{D}{\bar{a}_{\bar{N} \mid}},
$$

where $\bar{a}_{\bar{N} \mid}=1-e^{-\xi N} / \xi, \xi$ is a continuous technical rate.

During the period after retirement $t \in[T, T+N]$, the insurer also invests in one risk-free asset and a risk asset. In addition, he has to pay the guaranteed annuity to pension members. The evolution of the pension fund during $[T, T+N]$ is described by the following equation:

$$
\mathrm{d} X(t)=[X(t)(r+\pi \lambda L(t))-\zeta] \mathrm{d} t+X(t) \pi \sqrt{L(t)} \mathrm{d} W_{1}(t)
$$

The objective of the optimization problem for a meanvariance pension management could be described as follows:

$$
\sup _{\pi \in \prod}\left\{\mathrm{E}_{t, x, l}\left[X^{\pi}(T+N)\right]-\operatorname{Var}_{t, x, l}\left[X^{\pi}(T+N)\right]\right\}
$$

where $\prod=\{\pi \mid \pi \in[0, \infty)\}$, which means that a short sell of the bonds is permitted.

\section{The Time Consistent Solution in the Framework of Mean-Variance Criterion}

3.1. The Accumulation Phase before Retirement. According to the recent research paper, such as Bjork and Murgoci [10] and so forth, the mean-variance optimal control problem is equivalent to the following Markovian time inconsistent stochastic optimal control problem:

$$
\begin{aligned}
& J(t, x, l, \pi)=\mathrm{E}_{t, x, l}\left[X^{\pi}(T)\right]-\frac{\gamma}{2} \operatorname{Var}_{t, x, l}\left[X^{\pi}(T)\right], \\
& =\mathrm{E}_{t, x, l}\left[X^{\pi}(T)\right]-\frac{\gamma}{2}\left\{\mathrm{E}_{t, x, l}\left[X^{\pi}(T)^{2}\right]\right. \\
& \left.-\left(\mathrm{E}_{t, x, l}\left[X^{\pi}(T)\right]\right)^{2}\right\} \\
& V(t, x, l)=\sup _{\pi \in \prod} J(t, x, l, \pi) .
\end{aligned}
$$

Denote

$$
\begin{aligned}
& y^{\pi}(t, x, l)=\mathrm{E}_{t, x, l}\left[X^{\pi}(T)\right], \\
& z^{\pi}(t, x, l)=\mathrm{E}_{t, x, l}\left[X^{\pi}(T)^{2}\right],
\end{aligned}
$$

and the value function

$$
V(t, x, l)=\sup _{\pi \in \prod}\left\{f\left(t, x, l, y^{\pi}(t, x, l), z^{\pi}(t, x, l)\right)\right\},
$$

where

$$
f(t, x, l, y, z)=y-\frac{\gamma}{2}\left(z-y^{2}\right) .
$$

Theorem 1 (verification theorem). If there exist three real functions $F, G, H:[0, T] \times R \times R \rightarrow R$ satisfying the following extended $H J B$ equations:

$$
\begin{aligned}
\sup _{\pi}\left\{F_{t}\right. & -f_{t}+\left(F_{x}-f_{x}\right)\left[r x+\lambda l x \pi+P \frac{\omega-\omega_{0}-2 t}{\omega-\omega_{0}-t}\right] \\
& +\left(F_{l}-f_{l}\right) k(\theta-l)+\frac{1}{2}\left(F_{x x}-U_{x x}^{\pi}\right) x^{2} l \pi^{2} \\
& \left.+\frac{1}{2}\left(F_{l l}-U_{l l}^{\pi}\right) \sigma^{2} l+\left(F_{x l}-U_{x l}^{\pi}\right) \rho \sigma x l \pi\right\}=0, \\
F(T, x, l) & =f\left(T, x, l, x, x^{2}\right),
\end{aligned}
$$

where

$$
\begin{aligned}
& G_{t}+G_{x}\left[r x+\lambda l x \pi+P \frac{\omega-\omega_{0}-2 t}{\omega-\omega_{0}-t}\right]+k(\theta-l) G_{l} \\
& \quad+\frac{1}{2} x^{2} l \pi^{2} G_{x x}+\frac{1}{2} \sigma^{2} l G_{l l}+\rho \sigma x l \pi G_{x l}=0 \\
& G(T, x, l)=x
\end{aligned}
$$

$$
\begin{aligned}
H_{t} & +H_{x}\left[r x+\lambda l x \pi+P \frac{\omega-\omega_{0}-2 t}{\omega-\omega_{0}-t}\right]+k(\theta-l) H_{l} \\
& +\frac{1}{2} x^{2} l \pi^{2} H_{x x}+\frac{1}{2} \sigma^{2} l H_{l l}+\rho \sigma x l \pi H_{x l}=0, \\
H & (T, x, l)=x^{2}, \\
U_{x x}^{\pi}:= & f_{x x}+2 f_{x y} y_{x}^{\pi}+2 f_{x z} z_{x}^{\pi}+f_{y y}\left(y_{x}^{\pi}\right)^{2} \\
& +f_{z z}\left(z_{x}^{\pi}\right)^{2}+2 f_{y z} y_{x}^{\pi} z_{x}^{\pi}, \\
U_{l l}^{\pi}:= & f_{l l}+2 f_{y l} y_{l}^{\pi}+2 f_{z l} z_{l}^{\pi}+f_{y y}\left(y_{l}^{\pi}\right)^{2} \\
& +f_{z z}\left(z_{l}^{\pi}\right)^{2}+2 f_{y z} y_{l}^{\pi} z_{l}^{\pi}, \\
U_{x l}^{\pi}:= & f_{x l}+f_{x y} y_{l}^{\pi}+f_{x z} z_{l}^{\pi}+f_{y l} y_{x}^{\pi}+f_{z l} z_{x}^{\pi}+f_{y y} y_{x}^{\pi} y_{l}^{\pi} \\
& +f_{y z} y_{x}^{\pi} z_{l}^{\pi}+f_{y z} y_{l}^{\pi} z_{x}^{\pi}+f_{z z} z_{x}^{\pi} z_{l}^{\pi} .
\end{aligned}
$$

Then $V(t, x, l)=F(t, x, l), y^{\pi^{*}}(t, x, l)=G(t, x, l), z^{\pi^{*}}(t, x, l)=$ $H(t, x, l)$ for the optimal investment strategy $\pi^{*}$. 
Proof. The way to prove the theorem is completely similar to Li et al. [8], so we omit the details here.

Theorem 2. For the optimal control problem (17), there exist unique optimal time-consistent strategy

$$
\pi^{*}=\frac{\lambda}{x \gamma e^{r(T-t)}} \cdot\left[1-\rho \sigma \lambda \frac{\left(1-e^{(t-T)(k+\lambda \rho \sigma)}\right)}{k+\lambda \rho \sigma}\right]
$$

and the optimal value function

$$
F(t, x, l)=e^{r(T-t)} x+\frac{B(t)}{\gamma}+\frac{C(t)}{\gamma},
$$

where $B(t)$ and $C(t)$ are given by (40) and (41) explicitly. $\gamma>0$ denotes the risk aversion coefficient.

Proof. According to (20), we have

$$
\begin{gathered}
f_{t}=f_{x}=f_{l}=f_{x x}=f_{x y}=f_{x z}=f_{y z} \\
=f_{x l}=f_{y l}=f_{z l}=f_{z z}=f_{l l}=0, \\
f_{y}=1+\gamma y, \quad f_{y y}=\gamma, \quad f_{z}=-\frac{\gamma}{2} .
\end{gathered}
$$

Plugging (26) into $U_{x x}^{\pi}, U_{l x}^{\pi}, U_{l l}^{\pi}$, respectively,

$$
U_{x x}^{\pi^{*}}=\gamma G_{x}^{2}, \quad U_{l l}^{\pi^{*}}=\gamma G_{l}^{2}, \quad U_{x l}^{\pi^{*}}=\gamma G_{x} G_{l} .
$$

According to (21)

$$
\begin{aligned}
\pi^{*} & =-\frac{\lambda\left(F_{x}-f_{x}\right)+\rho \sigma\left(F_{x l}-U_{x l}^{\pi^{*}}\right)}{x\left(F_{x x}-U_{x x}^{\pi^{*}}\right)} \\
& =-\frac{\lambda F_{x}+\rho \sigma\left(F_{x l}-\gamma G_{x} G_{l}\right)}{x\left(F_{x x}-\gamma G_{x}^{2}\right)} .
\end{aligned}
$$

Equation (21) turns into

$$
\begin{aligned}
& F_{t}+F_{x}\left[r x+P \frac{\omega-\omega_{0}-2 t}{\omega-\omega_{0}-t}\right]+k(\theta-l) F_{l} \\
& \quad+\frac{1}{2}\left(F_{l l}-\gamma G_{l}^{2}\right) \sigma^{2} l-\frac{l}{2} \frac{\left[\lambda F_{x}+\rho \sigma\left(F_{x l}-\gamma G_{x} G_{l}\right)\right]^{2}}{F_{x x}-\gamma G_{x}^{2}}=0, \\
& F(T, x, l)=f\left(T, x, l, x, x^{2}\right),
\end{aligned}
$$

and (22) becomes

$$
\begin{aligned}
& G_{t}+G_{x}\left[r x+P \frac{\omega-\omega_{0}-2 t}{\omega-\omega_{0}-t}\right]+k(\theta-l) G_{l}+\frac{1}{2} \sigma^{2} l G_{l l} \\
&-\left(\lambda l G_{x}+\rho \sigma l G_{x l}\right)\left[\frac{\lambda F_{x}+\rho \sigma\left(F_{x l}-\gamma G_{x} G_{l}\right)}{F_{x x}-\gamma G_{x}^{2}}\right] \\
&+\frac{1}{2} l G_{x x}\left[\frac{\lambda F_{x}+\rho \sigma\left(F_{x l}-\gamma G_{x} G_{l}\right)}{F_{x x}-\gamma G_{x}^{2}}\right]^{2}=0, \\
& G(T, x, l)=x .
\end{aligned}
$$

The remainder of this section focuses on solving (29) w.r.t. $F$ and (30) w.r.t. $G$. Since the two equations are linear in $x$ and $l$, it is quite natural to conjecture the following forms of $F(t, x, l)$ and $G(t, x, l)$ :

$$
\begin{aligned}
& F(t, x, l)=A(t) x+\frac{B(t)}{\gamma} l+\frac{C(t)}{\gamma}, \\
& A(T)=1, \quad B(T)=0, \quad C(T)=0, \\
& G(t, x, l)=\alpha(t) x+\frac{\beta(t)}{\gamma} l+\frac{\Delta(t)}{\gamma}, \\
& \alpha(T)=1, \quad \beta(T)=0, \quad \Delta(T)=0,
\end{aligned}
$$

and the corresponding partial derivatives are

$$
\begin{array}{lll}
F_{t}=A_{t} x+\frac{B_{t}}{\gamma} l+\frac{C_{t}}{\gamma}, & F_{x}=A(t), & F_{l}=\frac{B(t)}{\gamma}, \\
F_{x x}=0, & F_{l l}=0, & F_{x l}=0, \\
G_{t}=\alpha_{t} x+\frac{\beta_{t}}{\gamma} l+\frac{\Delta_{t}}{\gamma}, & G_{x}=\alpha(t), & G_{l}=\frac{\beta(t)}{\gamma}, \\
G_{x x}=0, \quad G_{l l}=0, & G_{x l}=0 .
\end{array}
$$

Plugging the above partial derivatives (32) correspondingly into (29), (30), and (28), we obtain

$$
\begin{aligned}
& A_{t} x+\frac{B_{t}}{\gamma} l+\frac{C_{t}}{\gamma}+A(t)\left[r x+P \frac{\omega-\omega_{0}-2 t}{\omega-\omega_{0}-t}\right] \\
& +k(\theta-l) \frac{B(t)}{\gamma}-\frac{\sigma^{2} l}{2 \gamma} \beta^{2}(t) \\
& +\frac{l[\lambda A(t)-\rho \sigma \alpha(t) \beta(t)]^{2}}{2 \gamma \alpha^{2}(t)}=0, \\
& A(T)=1, \quad B(T)=C(T)=0, \\
& \alpha_{t} x+\frac{\beta_{t}}{\gamma} l+\frac{\Delta_{t}}{\gamma}+\alpha(t)\left[r x+P \frac{\omega-\omega_{0}-2 t}{\omega-\omega_{0}-t}\right] \\
& +k(\theta-l) \frac{\beta(t)}{\gamma}+\frac{\lambda l[\lambda A(t)-\rho \sigma \alpha(t) \beta(t)]}{\gamma \alpha(t)}=0, \\
& \quad \alpha(T)=1, \quad \beta(T)=\Delta(T)=0,
\end{aligned}
$$

and the optimal strategy

$$
\begin{aligned}
\pi^{*} & =-\frac{\lambda F_{x}+\rho \sigma\left(-\gamma G_{x} G_{l}\right)}{x\left(-\gamma G_{x}^{2}\right)}=\frac{\lambda F_{x}-\rho \sigma \gamma G_{x} G_{l}}{x \gamma G_{x}^{2}} \\
& =\frac{\lambda A(t)}{x \gamma \alpha^{2}(t)}-\frac{\rho \sigma \beta(t)}{x \gamma \alpha(t)} .
\end{aligned}
$$


Equation (33) splits into three equations:

$\left[A_{t}+r A(t)\right] x=0, \quad A(T)=1$,

$\left[\frac{B_{t}}{\gamma}-\frac{k B(t)}{\gamma}+\frac{\lambda^{2}}{2 \gamma} \frac{A^{2}(t)}{\alpha^{2}(t)}-\frac{\sigma^{2} \beta^{2}(t)}{2 \gamma}+\frac{\rho^{2} \sigma^{2} \beta^{2}(t)}{2 \gamma}\right.$

$\left.-\frac{\rho \sigma \lambda A(t) \beta(t)}{\gamma \alpha(t)}\right] l=0, \quad B(T)=0$,

$\frac{C_{t}}{\gamma}+A(t) P \frac{\omega-\omega_{0}-2 t}{\omega-\omega_{0}-t}+\frac{k \theta B(t)}{\gamma}=0, \quad C(T)=0$.

Equation (34) splits into three equations:

$$
\begin{gathered}
{\left[\alpha_{t}+r \alpha(t)\right] x=0, \quad \alpha(T)=1,} \\
{\left[\frac{\beta_{t}}{\gamma}-\frac{k \beta(t)}{\gamma}+\frac{\lambda^{2}}{\gamma} \frac{A(t)}{\alpha(t)}-\frac{\rho \sigma \lambda}{\gamma} \beta(t)\right] l=0, \quad \beta(T)=0,} \\
\frac{\Delta_{t}}{\gamma}+\alpha(t) P \frac{\omega-\omega_{0}-2 t}{\omega-\omega_{0}-t}+\frac{k \theta \beta(t)}{\gamma}=0, \quad \Delta(T)=0 .
\end{gathered}
$$

To solve the above equations, we have

$$
\begin{gathered}
A(t)=\alpha(t)=e^{r(T-t)}, \\
\beta(t)=\frac{\left(1-e^{(t-T)(k+\lambda \rho \sigma)}\right) \lambda^{2}}{k+\lambda \rho \sigma},
\end{gathered}
$$

$B(t)$

$$
\begin{gathered}
=\left[2 k \rho(k+\lambda \rho \sigma)^{2}(k+2 \lambda \rho \sigma)\right]^{-1} \cdot e^{-k T} \lambda^{2} \\
\cdot\left[-e^{k(2 t-T)+2(t-T) \lambda \rho \sigma} k \lambda^{2} \rho\left(-1+\rho^{2}\right) \sigma^{2}\right. \\
+e^{k t}(k \rho+2 \lambda \sigma)(k+\lambda \rho \sigma)^{2} \\
+e^{k T} \rho(k-\lambda \sigma)(k+\lambda \sigma)(k+2 \lambda \rho \sigma) \\
\left.-2 e^{k t+(t-T) \lambda \rho \sigma} k(k \rho+\lambda \sigma)(k+2 \lambda \rho \sigma)\right],
\end{gathered}
$$

$C(t)$

$$
\begin{aligned}
= & \frac{\left(-1+e^{r(-t+T)}\right) P \gamma}{r}+\left(2 \rho(k+\lambda \rho \sigma)^{2}(k+2 \lambda \rho \sigma)\right)^{-1} \\
& \times e^{-k T} \theta \lambda^{2}
\end{aligned}
$$

$$
\begin{aligned}
& \cdot\left[-\frac{\left(e^{k T}-e^{k(2 t-T)+2(t-T) \lambda \rho \sigma}\right) k \lambda^{2} \rho\left(-1+\rho^{2}\right) \sigma^{2}}{2(k+\lambda \rho \sigma)}\right. \\
& +\frac{\left(-e^{k t}+e^{k T}\right)(k \rho+2 \lambda \sigma)(k+\lambda \rho \sigma)^{2}}{k} \\
& +e^{k T}(-t+T) \rho(k-\lambda \sigma)(k+\lambda \sigma)(k+2 \lambda \rho \sigma) \\
& \left.+\frac{2\left(-e^{k T}+e^{k t+(t-T) \lambda \rho \sigma}\right) k(k \rho+\lambda \sigma)(k+2 \lambda \rho \sigma)}{k+\lambda \rho \sigma}\right] \\
& -\int_{t}^{T} \gamma p \frac{\tau}{\omega-\omega_{0}-\tau} e^{r(T-\tau)} \mathrm{d} \tau,
\end{aligned}
$$

$\Delta(t)$

$$
\begin{gathered}
=\frac{\left(-1+e^{r(-t+T)}\right) P \gamma}{r}-\int_{t}^{T} \gamma p \frac{\tau}{\omega-\omega_{0}-\tau} e^{r(T-\tau)} \mathrm{d} \tau \\
+\frac{k \theta \lambda^{2}\left(-1+e^{(t-T)(k+\lambda \rho \sigma)}+(-t+T)(k+\lambda \rho \sigma)\right)}{(k+\lambda \rho \sigma)^{2}} .
\end{gathered}
$$

After some simple calculations, the optimal investment strategy (35) becomes

$$
\begin{gathered}
\pi^{*}=\frac{1}{x \gamma} \cdot[\alpha(t)]^{-1} \cdot[\lambda-\rho \sigma \beta(t)] \\
=\frac{\lambda}{x \gamma e^{r(T-t)}} \cdot\left[1-\rho \sigma \lambda \frac{\left(1-e^{(t-T)(k+\lambda \rho \sigma)}\right)}{k+\lambda \rho \sigma}\right], \\
G(t, x, l)=e^{r(T-t)} x+\frac{\left(1-e^{(t-T)(k+\lambda \rho \sigma)}\right) \lambda^{2}}{\gamma(k+\lambda \rho \sigma)} l+\frac{\Delta(t)}{\gamma}, \\
F(t, x, l)=e^{r(T-t)} x+\frac{B(t)}{\gamma} l+\frac{C(t)}{\gamma} .
\end{gathered}
$$

Theorems 1 and 2 imply that

$$
\begin{gathered}
F(t, x, l)=V(t, x, l)=f\left(t, x, l, y^{\pi^{*}}, z^{\pi^{*}}\right) \\
=\mathrm{E}_{t, x, l}\left[X^{\pi}(T)\right]-\frac{\gamma}{2}\left\{\mathrm{E}_{t, x, l}\left[X^{\pi}(T)^{2}\right]\right. \\
\left.-\left(\mathrm{E}_{t, x, l}\left[X^{\pi}(T)\right]\right)^{2}\right\} \\
=G(t, x, l)-\frac{\gamma}{2}\left[H(t, x, l)-G(t, x, l)^{2}\right], \\
\mathrm{E}_{t, x, l}\left[X^{\pi^{*}}(T)\right]=G(t, x, l) .
\end{gathered}
$$

Since

$\operatorname{Var}_{t, x, l}\left[X^{\pi^{*}}(T)\right]=\mathrm{E}_{t, x, l}\left[X^{\pi^{*}}(T)\right]^{2}-\left(\mathrm{E}_{t, x, l} X^{\pi^{*}}(T)\right)^{2}$, 
(44) and (46) imply that

$$
\begin{aligned}
\operatorname{Var}_{t, x, l}\left[X^{\pi^{*}}(T)\right] \\
=\frac{2}{\gamma}[G(t, x, l)-F(t, x, l)] \\
=\frac{2}{\gamma^{2}}[(\beta(t)-B(t)) l+(\Delta(t)-C(t))] \\
=\left(2 k \gamma^{2} \rho(k+\lambda \rho \sigma)^{3}(k+2 \lambda \rho \sigma)\right)^{-1} e^{-k T} \lambda^{2} \cdot \Upsilon,
\end{aligned}
$$

where

$$
\begin{aligned}
\Upsilon=\{ & 2 e^{k t}(l-\theta)(k \rho+2 \lambda \sigma)(k+\lambda \rho \sigma)^{3} \\
& -4 e^{k t+(t-T) \lambda \rho \sigma} k \lambda\left(-1+\rho^{2}\right) \sigma(k+2 \lambda \rho \sigma) \\
& \times(k(l-\theta)+l \lambda \rho \sigma) \\
& +e^{k(2 t-T)+2(t-T) \lambda \rho \sigma} k \lambda^{2} \rho\left(-1+\rho^{2}\right) \sigma^{2} \\
& \times(2 k l-k \theta+2 l \lambda \rho \sigma) \\
& +e^{k T} \rho(k+2 \lambda \rho \sigma) \\
& \times\left[2 k^{3}(l+(-1-k t+k T) \theta)\right. \\
& +6 k^{2}(l+(-1-k t+k T) \theta) \lambda \rho \sigma \\
& +k \lambda^{2}\left(l\left(2+4 \rho^{2}\right)\right. \\
& \left.\quad-\theta\left(5+\rho^{2}+2 k(t-T)\left(1+2 \rho^{2}\right)\right)\right) \sigma^{2} \\
& \left.\left.+2(l+(-1-k t+k T) \theta) \lambda^{3} \rho \sigma^{3}\right]\right\} .
\end{aligned}
$$

Putting (45) and (47) together, the efficient frontier is rewritten as

$$
\begin{aligned}
\mathrm{E}_{t, x, l}\left[X^{\pi^{*}}(T)\right] & \\
= & e^{r(T-t)} x+\frac{\left(1-e^{(t-T)(k+\lambda \rho \sigma)}\right) \lambda^{2}}{\gamma(k+\lambda \rho \sigma)} l+\frac{\Delta(t)}{\gamma} \\
& +\gamma \sqrt{\operatorname{Var}_{t, x, l}\left[X^{\pi^{*}}(T)\right]} \\
& -\sqrt{2[(\beta(t)-B(t)) l+(\Delta(t)-C(t))]},
\end{aligned}
$$

where $(t), B(t), C(t)$, and $\Delta(t)$ are given by (39), (40), (41), and (42), respectively.

3.2. The Distribution Phase after Retirement. According to the recent research paper, such as Bjork and Murgoci [10] and so forth, the mean-variance optimal control problem in the distribution phase is equivalent to the following Markovian time inconsistent stochastic optimal control problem:

$$
\begin{gathered}
\widehat{J}(t, x, l, \pi) \\
=\mathrm{E}_{t, x, l}\left[X^{\pi}(T+N)\right]-\frac{\gamma}{2} \operatorname{Var}_{t, x, l}\left[X^{\pi}(T+N)\right], \\
=\mathrm{E}_{t, x, l}\left[X^{\pi}(T+N)\right]-\frac{\gamma}{2}\left\{\mathrm{E}_{t, x, l}\left[X^{\pi}(T+N)^{2}\right]\right. \\
\left.-\left(\mathrm{E}_{t, x, l}\left[X^{\pi}(T+N)\right]\right)^{2}\right\}, \\
\widehat{V}(t, x, l)=\sup _{\pi \in \prod} \widehat{J}(t, x, l, \pi) .
\end{gathered}
$$

Denote

$$
\begin{aligned}
& y^{\pi}(t, x, l)=\mathrm{E}_{t, x, l}\left[X^{\pi}(T+N)\right], \\
& z^{\pi}(t, x, l)=\mathrm{E}_{t, x, l}\left[X^{\pi}(T+N)^{2}\right],
\end{aligned}
$$

and the value function

$$
\widehat{V}(t, x, l)=\sup _{\pi \in \prod}\left\{\widehat{f}\left(t, x, l, y^{\pi}(t, x, l), z^{\pi}(t, x, l)\right)\right\},
$$

where

$$
\widehat{f}(t, x, l, y, z)=y-\frac{\gamma}{2}\left(z-y^{2}\right) .
$$

Theorem 3 (verification theorem). If there exist three real functions $\widehat{F}, \widehat{G}, \widehat{H}:[0, T] \times R \times R \rightarrow R$ satisfying the following extended $H J B$ equations:

$$
\begin{aligned}
\sup _{\pi}\left\{\widehat{F}_{t}-\widehat{f}_{t}+\left(\widehat{F}_{x}-\widehat{f}_{x}\right)[r x+\lambda l x \pi-\zeta]\right. \\
+\left(\widehat{F}_{l}-\widehat{f}_{l}\right) k(\theta-l)+\frac{1}{2}\left(\widehat{F}_{x x}-\widehat{U}_{x x}^{\pi}\right) x^{2} l \pi^{2} \\
\left.\quad+\frac{1}{2}\left(\widehat{F}_{l l}-\widehat{U}_{l l}^{\pi}\right) \sigma^{2} l+\left(\widehat{F}_{x l}-\widehat{U}_{x l}^{\pi}\right) \rho \sigma x l \pi\right\}=0, \\
\widehat{F}(T+N, x, l)=\widehat{f}\left(T+N, x, l, x, x^{2}\right),
\end{aligned}
$$

where

$$
\begin{aligned}
\widehat{G}_{t} & +\widehat{G}_{x}[r x+\lambda l x \pi-\zeta]+k(\theta-l) \widehat{G}_{l} \\
& +\frac{1}{2} x^{2} l \pi^{2} \widehat{G}_{x x}+\frac{1}{2} \sigma^{2} l \widehat{G}_{l l}+\rho \sigma x l \pi \widehat{G}_{x l}=0, \\
\widehat{G}(T+N, x, l)=x & \\
\widehat{H}_{t} & +\widehat{H}_{x}[r x+\lambda l x \pi-\zeta]+k(\theta-l) \widehat{H}_{l} \\
& +\frac{1}{2} x^{2} l \pi^{2} \widehat{H}_{x x}+\frac{1}{2} \sigma^{2} l \widehat{H}_{l l}+\rho \sigma x l \pi \widehat{H}_{x l}=0, \\
\widehat{H}(T+N, x, l)=x^{2} & \\
\widehat{U}_{x x}^{\pi}:= & \widehat{f}_{x x}+2 \widehat{f}_{x y} y_{x}^{\pi}+2 \widehat{f}_{x z} z_{x}^{\pi} \\
& +\widehat{f}_{y y}\left(y_{x}^{\pi}\right)^{2}+\widehat{f}_{z z}\left(z_{x}^{\pi}\right)^{2}+2 \widehat{f}_{y z} y_{x}^{\pi} z_{x}^{\pi},
\end{aligned}
$$




$$
\begin{aligned}
\widehat{U}_{l l}^{\pi}:= & \widehat{f}_{l l}+2 \widehat{f}_{y l} y_{l}^{\pi}+2 \widehat{f}_{z l} z_{l}^{\pi} \\
& +\widehat{f}_{y y}\left(y_{l}^{\pi}\right)^{2}+\widehat{f}_{z z}\left(z_{l}^{\pi}\right)^{2}+2 \widehat{f}_{y z} y_{l}^{\pi} z_{l}^{\pi}, \\
\widehat{U}_{x l}^{\pi}:= & \widehat{f}_{x l}+\widehat{f}_{x y} y_{l}^{\pi}+\widehat{f}_{x z} z_{l}^{\pi}+\widehat{f}_{y l} y_{x}^{\pi}+\widehat{f}_{z l} z_{x}^{\pi} \\
& +\widehat{f}_{y y} y_{x}^{\pi} y_{l}^{\pi}+\widehat{f}_{y z} y_{x}^{\pi} z_{l}^{\pi}+\widehat{f}_{y z} y_{l}^{\pi} z_{x}^{\pi}+\widehat{f}_{z z} z_{x}^{\pi} z_{l}^{\pi} .
\end{aligned}
$$

Then $\widehat{V}(t, x, l)=\widehat{F}(t, x, l), y^{\pi^{*}}(t, x, l)=\widehat{G}(t, x, l), z^{\pi^{*}}(t, x, l)=$ $\widehat{H}(t, x, l)$ for the optimal investment strategy $\pi^{*}$.

Proof. The way to prove the theorem is completely similar to Li et al. [8], so we omit the details here.

Theorem 4. For the optimal control problem (50), there exist unique optimal time-consistent strategy

$$
\pi^{*}=\frac{\lambda}{x \gamma e^{r(N+T-t)}} \cdot\left[1-\rho \sigma \lambda \frac{\left(1-e^{-(N-t+T)(k+\lambda \rho \sigma)}\right)}{k+\lambda \rho \sigma}\right]
$$

and the optimal value function

$$
\widehat{F}(t, x, l)=e^{r(N+T-t)} x+\frac{\widehat{B}(t)}{\gamma}+\frac{\widehat{C}(t)}{\gamma},
$$

where $\widehat{B}(t)$ and $\widehat{C}(t)$ are given by (72) and (74), explicitly. $\gamma>0$ denotes the risk aversion coefficient.

Proof. According to (53), we have

$$
\begin{aligned}
\widehat{f}_{t} & =\widehat{f}_{x}=\widehat{f}_{l}=\widehat{f}_{x x}=\widehat{f}_{x y}=\widehat{f}_{x z}=\widehat{f}_{y z} \\
& =\widehat{f}_{x l}=\widehat{f}_{y l}=\widehat{f}_{z l}=\widehat{f}_{z z}=\widehat{f}_{l l}=0, \\
\widehat{f}_{y} & =1+\gamma y, \quad \widehat{f}_{y y}=\gamma, \quad \widehat{f}_{z}=-\frac{\gamma}{2} .
\end{aligned}
$$

Substituting (59) into $\widehat{U}_{x x}^{\pi}, \widehat{U}_{l x}^{\pi}, \widehat{U}_{l l}^{\pi}$

$$
\widehat{U}_{x x}^{\pi^{*}}=\gamma \widehat{G}_{x}^{2}, \quad \widehat{U}_{l l}^{\pi^{*}}=\gamma \widehat{G}_{l}^{2}, \quad \widehat{U}_{x l}^{\pi^{*}}=\gamma \widehat{G}_{x} \widehat{G}_{l} .
$$

Taking derivative for (54) with respect to $\pi$, according to the first-order necessary condition, we have

$$
\left(\widehat{F}_{x}-\widehat{f}_{x}\right) \lambda l x+\left(\widehat{F}_{x x}-\widehat{U}_{x x}^{\pi}\right) x^{2} l \pi+\left(\widehat{F}_{x l}-\widehat{U}_{x l}^{\pi}\right) \rho \sigma x l=0,
$$

so

$$
\pi^{*}=-\frac{\left(\widehat{F}_{x}-\widehat{f}_{x}\right) \lambda+\left(\widehat{F}_{x l}-\widehat{U}_{x l}^{\pi}\right) \rho \sigma}{\left(\widehat{F}_{x x}-\widehat{U}_{x x}^{\pi}\right) x}=\frac{\lambda \widehat{F}_{x}-\rho \sigma \gamma \widehat{G}_{x} \widehat{G}_{l}}{\gamma x \widehat{G}_{x}^{2}} .
$$

The remainder of this section focuses on solving (54) w.r.t. $\widehat{F}$ and (55) w.r.t. $\widehat{G}$. Since the two equations are linear in $x$ and $l$, it is quite natural to conjecture the following forms of $\widehat{F}(t, x, l)$ and $\widehat{G}(t, x, l)$ :

$$
\begin{array}{ll}
\widehat{F}(t, x, l)=\widehat{A}(t) x+\frac{\widehat{B}(t)}{\gamma} l+\frac{\widehat{C}(t)}{\gamma}, & \\
\widehat{A}(T+N)=1, \quad \widehat{B}(T+N)=0, & \widehat{C}(T+N)=0, \\
\widehat{G}(t, x, l)=\widehat{\alpha}(t) x+\frac{\widehat{\beta}(t)}{\gamma} l+\frac{\widehat{\Delta}(t)}{\gamma}, & \\
\widehat{\alpha}(T+N)=1, \quad \widehat{\beta}(T+N)=0, & \widehat{\Delta}(T+N)=0,
\end{array}
$$

and the corresponding partial derivatives are

$$
\begin{array}{ll}
\widehat{F}_{t}=\widehat{A}_{t} x+\frac{\widehat{B}_{t}}{\gamma} l+\frac{\widehat{C}_{t}}{\gamma}, & \widehat{F}_{x}=\widehat{A}(t), \\
\widehat{F}_{l}=\frac{\widehat{B}(t)}{\gamma}, \quad \widehat{F}_{x x}=0, & \widehat{F}_{l l}=0, \quad \widehat{F}_{x l}=0, \\
\widehat{G}_{t}=\widehat{\alpha}_{t} x+\frac{\widehat{\beta}_{t}}{\gamma} l+\frac{\widehat{\Delta}_{t}}{\gamma}, & \widehat{G}_{x}=\widehat{\alpha}(t),
\end{array}
$$

$\widehat{G}_{l}=\frac{\widehat{\beta}(t)}{\gamma}, \quad \widehat{G}_{x x}=0, \quad \widehat{G}_{l l}=0, \quad \widehat{G}_{x l}=0$.

Plugging the above partial derivatives (64) correspondingly into (54), (55), and (62), we obtain

$$
\begin{gathered}
\widehat{A}_{t} x+\frac{\widehat{B}_{t}}{\gamma} l+\frac{\widehat{C}_{t}}{\gamma}+\widehat{A}(t)[r x-\zeta]+k(\theta-l) \frac{\widehat{B}(t)}{\gamma} \\
-\frac{\sigma^{2} l}{2 \gamma} \widehat{\beta}^{2}(t)+\frac{l[\lambda \widehat{A}(t)-\rho \sigma \widehat{\alpha}(t) \widehat{\beta}(t)]^{2}}{2 \gamma \widehat{\alpha}^{2}(t)}=0, \\
\widehat{A}(T+N)=1, \quad \widehat{B}(T+N)=\widehat{C}(T+N)=0, \\
\widehat{\alpha}_{t} x+\frac{\widehat{\beta}_{t}}{\gamma} l+\frac{\widehat{\Delta}_{t}}{\gamma}+\widehat{\alpha}(t)[r x-\zeta]+k(\theta-l) \frac{\widehat{\beta}(t)}{\gamma} \\
+\frac{\lambda l[\lambda \widehat{A}(t)-\rho \sigma \widehat{\alpha}(t) \widehat{\beta}(t)]}{\gamma \widehat{\alpha}(t)}=0, \\
\widehat{\alpha}(T+N)=1, \quad \widehat{\beta}(T+N)=\widehat{\Delta}(T+N)=0,
\end{gathered}
$$

and the optimal strategy

$$
\begin{aligned}
\pi^{*} & =-\frac{\lambda \widehat{F}_{x}+\rho \sigma\left(-\gamma \widehat{G}_{x} \widehat{G}_{l}\right)}{x\left(-\gamma \widehat{G}_{x}^{2}\right)}=\frac{\lambda \widehat{F}_{x}-\rho \sigma \gamma \widehat{G}_{x} \widehat{G}_{l}}{x \gamma \widehat{G}_{x}^{2}} \\
& =\frac{\lambda \widehat{A}(t)}{x \gamma \widehat{\alpha}^{2}(t)}-\frac{\rho \sigma \widehat{\beta}(t)}{x \gamma \widehat{\alpha}(t)} .
\end{aligned}
$$


Equation (65) splits into three equations

$$
\begin{aligned}
& {\left[\widehat{A}_{t}+r \widehat{A}(t)\right] x=0, \quad \widehat{A}(T+N)=1,} \\
& {\left[\frac{\widehat{B}_{t}}{\gamma}-\frac{k \widehat{B}(t)}{\gamma}+\frac{\lambda^{2}}{2 \gamma} \frac{\widehat{A}^{2}(t)}{\widehat{\alpha}^{2}(t)}-\frac{\sigma^{2} \widehat{\beta}^{2}(t)}{2 \gamma}+\frac{\rho^{2} \sigma^{2} \widehat{\beta}^{2}(t)}{2 \gamma}\right.} \\
& \left.\quad-\frac{\rho \sigma \lambda \widehat{A}(t) \widehat{\beta}(t)}{\gamma \widehat{\alpha}(t)}\right] l=0, \quad \widehat{B}(T+N)=0, \\
& \frac{\widehat{C}_{t}}{\gamma}-\zeta \widehat{A}(t)+\frac{k \theta \widehat{B}(t)}{\gamma}=0, \quad \widehat{C}(T+N)=0 .
\end{aligned}
$$

Equation (66) splits into three equations

$$
\begin{aligned}
& {\left[\widehat{\alpha}_{t}+r \widehat{\alpha}(t)\right] x=0, \quad \widehat{\alpha}(T+N)=1,} \\
& {\left[\frac{\widehat{\beta}_{t}}{\gamma}-\frac{k \widehat{\beta}(t)}{\gamma}+\frac{\lambda^{2}}{\gamma} \frac{\widehat{A}(t)}{\widehat{\alpha}(t)}-\frac{\rho \sigma \lambda}{\gamma} \widehat{\beta}(t)\right] l=0,} \\
& \widehat{\beta}(T+N)=0, \\
& \frac{\widehat{\Delta}_{t}}{\gamma}-\zeta \widehat{\alpha}(t)+\frac{k \theta \hat{\beta}(t)}{\gamma}=0, \quad \widehat{\Delta}(T+N)=0 .
\end{aligned}
$$

To solve the above equations, we have

$$
\begin{gathered}
\widehat{A}(t)=\widehat{\alpha}(t)=e^{r(N+T-t)}, \\
\widehat{\beta}(t)=\frac{\left(1-e^{-(N-t+T)(k+\lambda \rho \sigma)}\right) \lambda^{2}}{k+\lambda \rho \sigma},
\end{gathered}
$$

$\widehat{B}(t)$

$$
\begin{aligned}
=(2 & \left.k \rho(k+\lambda \rho \sigma)^{2}(k+2 \lambda \rho \sigma)\right)^{-1} \\
\times & e^{-k(3 N+2 t+3 T)-3(N+t+T) \lambda \rho \sigma} \lambda^{2} \\
\cdot & -e^{k(N+4 t+T)+(N+5 t+T) \lambda \rho \sigma} k \lambda^{2} \rho\left(-1+\rho^{2}\right) \sigma^{2} \\
& +e^{k(2 N+3 t+2 T)+3(N+t+T) \lambda \rho \sigma}(k \rho+2 \lambda \sigma) \\
& \times(k+\lambda \rho \sigma)^{2} \\
& +e^{k(3 N+2 t+3 T)+3(N+t+T) \lambda \rho \sigma} \rho(k-\lambda \sigma)(k+\lambda \sigma) \\
& \times(k+2 \lambda \rho \sigma)-2 e^{k(2 N+3 t+2 T)+2(N+2 t+T) \lambda \rho \sigma} \\
& \times k(k \rho+\lambda \sigma)(k+2 \lambda \rho \sigma)]
\end{aligned}
$$

$\widehat{\Delta}(t)$

$$
\begin{aligned}
& =\left(r(k+\lambda \rho \sigma)^{2}\right)^{-1} e^{-r t-(N+T)(k+\lambda \rho \sigma)} \\
& \cdot\left[e^{t(k+r+\lambda \rho \sigma)} k r \theta \lambda^{2}-e^{(N+T)(k+r+\lambda \rho \sigma)}\right. \\
& \times \gamma \zeta(k+\lambda \rho \sigma)^{2}+e^{r t+(N+T)(k+\lambda \rho \sigma)} \\
& \times\left[k\left(k \gamma \zeta+r(-1+k(N-t+T)) \theta \lambda^{2}\right)\right. \\
& +k \lambda\left(2 \gamma \zeta+r(N-t+T) \theta \lambda^{2}\right) \rho \sigma \\
& \left.\left.+\gamma \zeta \lambda^{2} \rho^{2} \sigma^{2}\right]\right],
\end{aligned}
$$

$\widehat{C}(t)$

$$
\begin{aligned}
& =\left(4 k r \rho(k+\lambda \rho \sigma)^{3}(k+2 \lambda \rho \sigma)\right)^{-1} \\
& \cdot\left\{-2 k^{5}\left(2\left(-1+e^{r(N-t+T)}\right) \gamma \zeta\right.\right. \\
& \left.-r(N-t+T) \theta \lambda^{2}\right) \rho \\
& +4 e^{-k(N-t+T)}\left(-1+e^{k(N-t+T)}\right) r \theta \lambda^{6} \rho^{3} \sigma^{4} \\
& +2 k^{4} \lambda \rho\left[-10\left(-1+e^{r(N-t+T)}\right) \gamma \zeta \rho \sigma\right. \\
& +r \theta \lambda\left(-1-e^{-k(N-t+T)}\right. \\
& +2 e^{-(N-t+T)(k+\lambda \rho \sigma)} \\
& +3(N-t+T) \lambda \rho \sigma)] \\
& -2 e^{-k(N-t+T)} k \lambda^{4} \rho^{2} \sigma^{3} \\
& \times\left[-4\left(e^{k(N-t+T)}-e^{(k+r)(N-t+T)}\right) \gamma \zeta \rho^{3} \sigma\right. \\
& +r \theta \lambda\left(6+\rho^{2}-e^{k(N-t+T)}\right. \\
& \left.\left.\times\left(6+\rho^{2}-2(N-t+T) \lambda \rho \sigma\right)\right)\right] \\
& +2 k^{3} \lambda^{2} \sigma \\
& \times\left[2 e^{-(N-t+T)(k+\lambda \rho \sigma)} r \theta \lambda\left(1+2 \rho^{2}\right)\right. \\
& -e^{-k(N-t+T)} r \theta \lambda\left(2+3 \rho^{2}\right) \\
& -18 e^{r(N-t+T)} \gamma \zeta \rho^{3} \sigma \\
& +\rho\left(18 \gamma \zeta \rho^{2} \sigma+r \theta \lambda\right. \\
& \left.\left.\times\left(-\rho+(N-t+T) \lambda\left(-1+2 \rho^{2}\right) \sigma\right)\right)\right] \\
& +e^{-(N-t+T)(3 k+2 \lambda \rho \sigma)} k^{2} \lambda^{3} \rho \sigma^{2} \\
& \times\left[8 e^{(N-t+T)(2 k+\lambda \rho \sigma)} r \theta \lambda\right.
\end{aligned}
$$




$$
\begin{aligned}
& -28 e^{(N-t+T)(3 k+r+2 \lambda \rho \sigma)} \gamma \zeta \rho^{3} \sigma \\
+ & e^{k(N-t+T)} r \theta \lambda\left(-1+\rho^{2}\right) \\
- & 6 e^{2(N-t+T)(k+\lambda \rho \sigma)} r \theta \lambda\left(2+\rho^{2}\right) \\
+ & e^{(N-t+T)(3 k+2 \lambda \rho \sigma)} \\
\times & \left(28 \gamma \zeta \rho^{3} \sigma+r \theta \lambda\right. \\
& \times(5+\rho(5 \rho-6(N-t+T) \lambda \sigma)))]\} .
\end{aligned}
$$

After simple calculation, the optimal investment strategy (67) becomes

$$
\begin{aligned}
& \pi^{*}=\frac{1}{x \gamma} \cdot[\widehat{\alpha}(t)]^{-1} \cdot[\lambda-\rho \sigma \widehat{\beta}(t)] \\
& =\frac{\lambda}{x \gamma e^{r(N-t+T)}} \cdot\left[1-\rho \sigma \lambda \frac{\left(1-e^{-(N-t+T)(k+\lambda \rho \sigma)}\right)}{k+\lambda \rho \sigma}\right] \text {, } \\
& \widehat{G}(t, x, l)=e^{r(N-t+T)} x+\frac{\left(1-e^{-(N-t+T)(k+\lambda \rho \sigma)}\right) \lambda^{2}}{\gamma(k+\lambda \rho \sigma)} l+\frac{\widehat{\Delta}(t)}{\gamma}, \\
& \widehat{F}(t, x, l)=e^{r(N-t+T)} x+\frac{\widehat{B}(t)}{\gamma} l+\frac{\widehat{C}(t)}{\gamma} .
\end{aligned}
$$

Theorem 3 and Theorem 4 imply that

$$
\begin{aligned}
\widehat{F}(t, x, l)= & \widehat{V}(t, x, l)=\widehat{f}\left(t, x, l, y^{\pi^{*}}, z^{\pi^{*}}\right) \\
= & \mathrm{E}_{t, x, l}\left[X^{\pi}(T+N)\right]-\frac{\gamma}{2} \\
& \times\left\{\mathrm{E}_{t, x, l}\left[X^{\pi}(T+N)^{2}\right]-\left(\mathrm{E}_{t, x, l}\left[X^{\pi}(T+N)\right]\right)^{2}\right\} \\
= & \widehat{G}(t, x, l)-\frac{\gamma}{2}\left[\widehat{H}(t, x, l)-\widehat{G}(t, x, l)^{2}\right], \\
& \mathrm{E}_{t, x, l}\left[X^{\pi^{*}}(T+N)\right]=\widehat{G}(t, x, l) .
\end{aligned}
$$

Since

$$
\begin{aligned}
\operatorname{Var}_{t, x, l} & {\left[X^{\pi^{*}}(T+N)\right] } \\
= & \mathrm{E}_{t, x, l}\left[X^{\pi^{*}}(T+N)\right]^{2}-\left(\mathrm{E}_{t, x, l} X^{\pi^{*}}(T+N)\right)^{2},
\end{aligned}
$$

(76) and (78) imply that

$$
\begin{aligned}
\operatorname{Var}_{t, x, l} & {\left[X^{\pi^{*}}(T+N)\right] } \\
= & \frac{2}{\gamma}[\widehat{G}(t, x, l)-\widehat{F}(t, x, l)] \\
= & \frac{2}{\gamma^{2}}[(\widehat{\beta}(t)-\widehat{B}(t)) l+(\widehat{\Delta}(t)-\widehat{C}(t))] \\
= & \left(2 k \gamma^{2} \rho(k+\lambda \rho \sigma)^{3}(k+2 \lambda \rho \sigma)\right)^{-1} \\
& \times e^{-2(N+T)(k+\lambda \rho \sigma)} \lambda^{2} \cdot \Theta,
\end{aligned}
$$

where

$$
\begin{gathered}
\Theta=\left\{-2 e^{k(N+t+T)+2(N+T) \lambda \rho \sigma}(l-\theta)(k \rho+2 \lambda \sigma)(k+\lambda \rho \sigma)^{3}\right. \\
-4 e^{(N+t+T)(k+\lambda \rho \sigma)} k \lambda\left(-1+\rho^{2}\right) \sigma(k+2 \lambda \rho \sigma) \\
\times(k(l-\theta)+l \lambda \rho \sigma) \\
+e^{2 t(k+\lambda \rho \sigma)} k \lambda^{2} \rho\left(-1+\rho^{2}\right) \sigma^{2}(2 k l-k \theta+2 l \lambda \rho \sigma) \\
+e^{2(N+T)(k+\lambda \rho \sigma)} \rho(k+2 \lambda \rho \sigma) \\
\times\left[2 k^{3}(l+(-1+k(N-t+T)) \theta)\right. \\
+6 k^{2}(l+(-1+k(N-t+T)) \theta) \lambda \rho \sigma \\
+2(l+(-1+k(N-t+T)) \theta) \lambda^{3} \rho \sigma^{3} \\
+k \lambda^{2}\left(l\left(2+4 \rho^{2}\right)\right. \\
+\theta\left(-5-\rho^{2}+2 k(N-t+T)\right. \\
\left.\left.\left.\left.\times\left(1+2 \rho^{2}\right)\right)\right) \sigma^{2}\right]\right\}
\end{gathered}
$$

Putting (77) and (79) together, the efficient frontier is rewritten as

$$
\begin{aligned}
\mathrm{E}_{t, x, l}\left[X^{\pi^{*}}(T+N)\right] & \\
= & e^{r(N-t+T)} x+\frac{\left(1-e^{-(N-t+T)(k+\lambda \rho \sigma)}\right) \lambda^{2}}{\gamma(k+\lambda \rho \sigma)} l+\frac{\widehat{\Delta}(t)}{\gamma} \\
& +\gamma \sqrt{\operatorname{Var}_{t, x, l}\left[X^{\pi^{*}}(T+N)\right]} \\
& -\sqrt{2[(\widehat{\beta}(t)-\widehat{B}(t)) l+(\widehat{\Delta}(t)-\widehat{C}(t))]},
\end{aligned}
$$

where $\widehat{\beta}(t), \widehat{B}(t), \widehat{\Delta}(t)$, and $\widehat{C}(t)$ are given by (71), (72), (73), and (74), respectively.

Remark 5. In a defined contribution pension plan, only the contributions in the accumulation phase before retirement are guaranteed, but the future benefits are undetermined. Considering a annuity contract in the distribution phase after retirement, it achieves the effect that both phases before and after retirement are "defined," which makes the pension plan even more portable and of great convenience for insurers.

\section{Numerical Analysis}

In this section, some numerical analysis and graphics are provided to illustrate our results. The main objectives are two aspects: one is to explain the properties of the optimal strategies derived in Section 2 and Section 3 and the other is to illustrate the efficient frontier.

First, let us analyze the expression of the optimal timeconsistent investment strategy $\pi^{*}$ in the accumulation phase before retirement. Analysis of the optimal time-consistent 


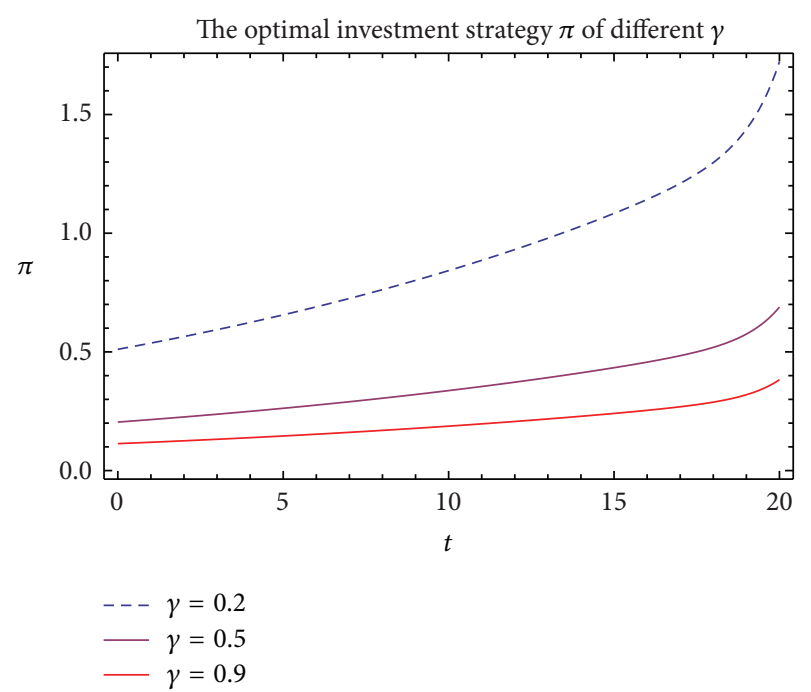

FIGURE 1: Evolutions of optimal investment strategy $\pi^{*}$ with different risk aversion coefficients $\gamma$.

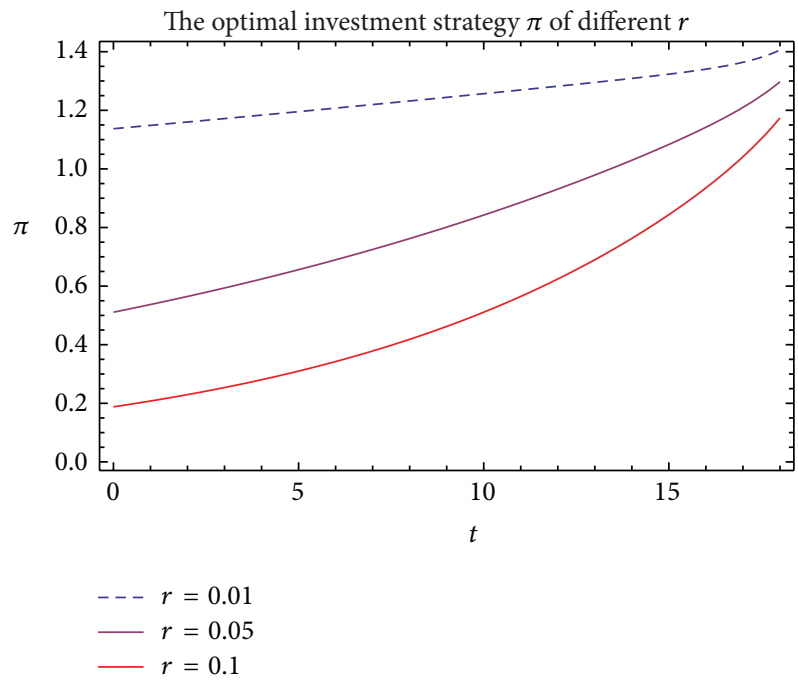

FIGURE 2: Evolutions of optimal investment strategy $\pi^{*}$ with different risk-free interest rates $r$.

investment strategy in the distribution phase after retirement is almost the same and we can get similar results, so it is omitted:

$$
\begin{aligned}
\pi^{*} & =\frac{\lambda}{x \gamma e^{r(T-t)}} \cdot\left[1-\rho \sigma \lambda \frac{\left(1-e^{(t-T)(k+\lambda \rho \sigma)}\right)}{k+\lambda \rho \sigma}\right] \\
& =\frac{\lambda e^{r(t-T)}}{x \gamma(k+\lambda \rho \sigma)} \cdot\left(k+\lambda \rho \sigma e^{(t-T)(k+\lambda \rho \sigma)}\right) .
\end{aligned}
$$

The derivative of (82) w.r.t. $\gamma$ is

$$
\frac{\partial \pi^{*}}{\partial \gamma}=-\frac{\lambda}{x \gamma^{2}} \cdot \frac{e^{r(t-T)}\left(k+e^{(t-T)(k+\lambda \rho \sigma)} \lambda \rho \sigma\right)}{(k+\lambda \rho \sigma)} .
$$

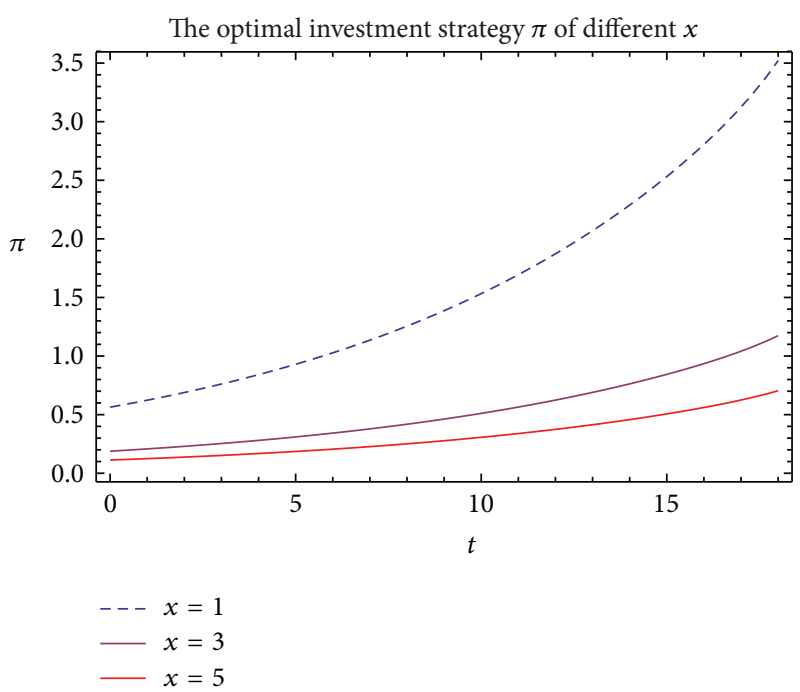

FIGURE 3: Evolutions of optimal investment strategy $\pi^{*}$ with different fund size $x$ for the same risk averse level.

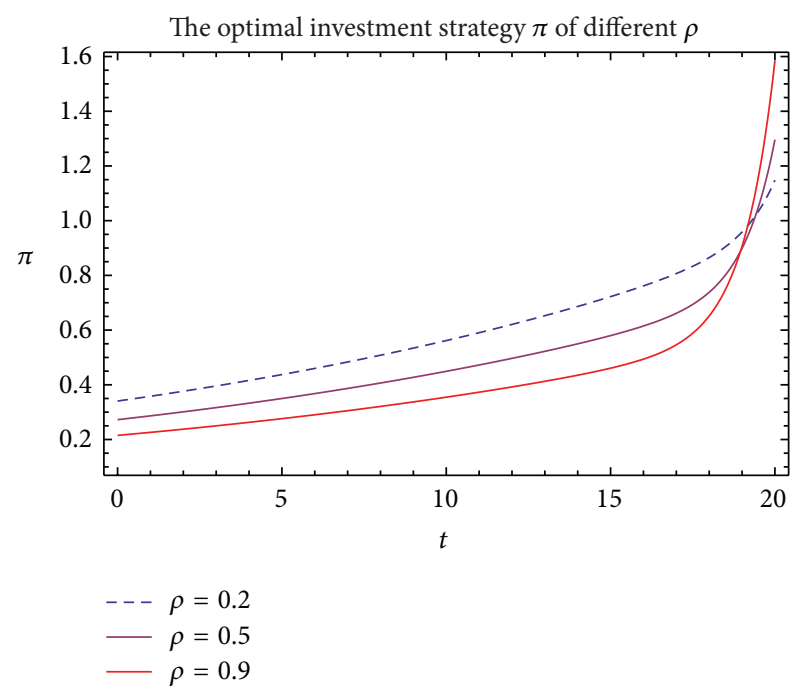

Figure 4: Evolutions of optimal investment strategy $\pi^{*}$ with different correlation coefficients $\rho$.

Since

$$
\frac{e^{r(t-T)}\left(k+e^{(t-T)(k+\lambda \rho \sigma)} \lambda \rho \sigma\right)}{(k+\lambda \rho \sigma)}>0, \quad \frac{\lambda}{x \gamma^{2}}>0,
$$

then

$$
\frac{\partial \pi^{*}}{\partial \gamma}<0
$$

which shows that the optimal investment policy decreases with respect to the risk aversion level $\gamma$, referring to Figure 1, which is consistent with reality. In fact, the higher degree of risk aversion, people should invest the less cash in risky assets to avoid risk. 


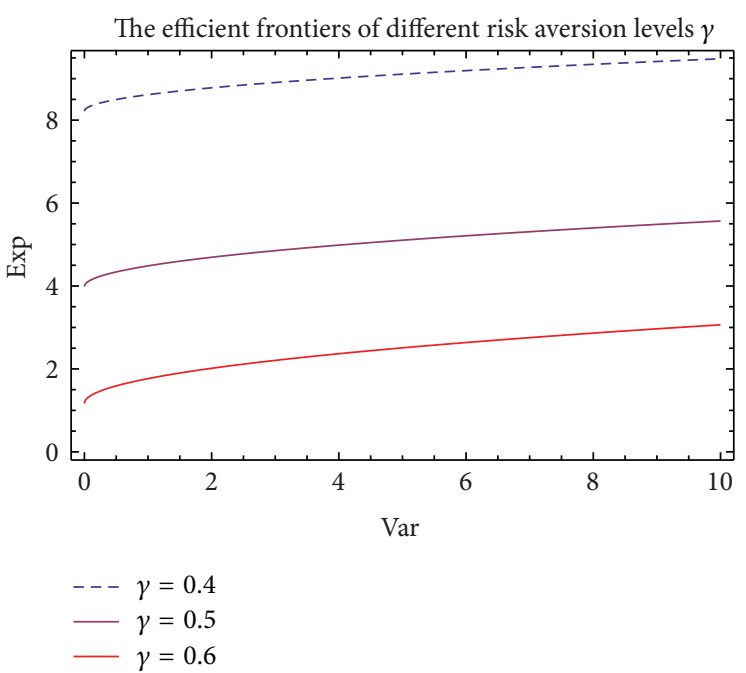

(a)

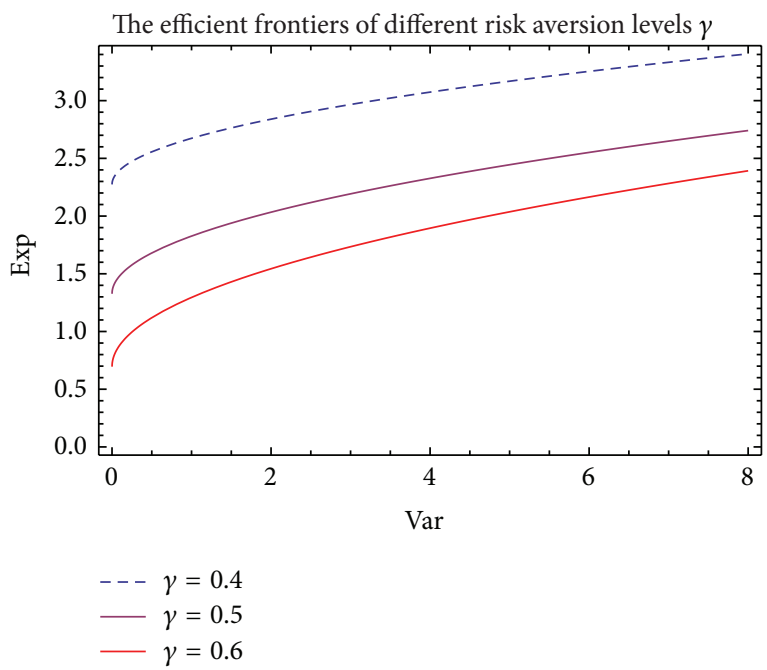

(b)

Figure 5: (a) The efficient frontiers of different risk averse level $\gamma$ when $t=21$. (b) The efficient frontiers of different risk averse level $\gamma$ when $t=28$.

Similarly, the derivatives of (82) w.r.t. risk-free interest rate $r$ and starting wealth level $x$ are given by

$$
\begin{gathered}
\frac{\partial \pi^{*}}{\partial r}=-\frac{e^{r(t-T)}(T-t) \lambda\left(k+e^{(t-T)} \lambda \rho \sigma(k+\lambda \rho \sigma)\right)}{x \gamma(k+\lambda \rho \sigma)}<0, \\
\frac{\partial \pi^{*}}{\partial x}=-\frac{e^{r(t-T)} \lambda\left(k+e^{(t-T)} \lambda \rho \sigma(k+\lambda \rho \sigma)\right)}{x^{2} \gamma(k+\lambda \rho \sigma)}<0,
\end{gathered}
$$

which mean that the optimal investment policy also decreases with respect to the risk-free interest rate $r$ and starting wealth level $x$. See Figures 2 and 3, respectively. Obviously, the higher the risk-free interest rate, people must increase the investment on the risk-free asset because they can get more profit without risk, and risk investment corresponds to reduction. Furthermore, a risk-averse investor must strive to control the investment amount of risk assets, if the initial capital size $x$ increasing, only by reducing the proportion $\pi$ can make the investment amount of risk assets stay at a relatively low level.

But for the derivative of (82) w.r.t. correlation coefficient $\rho$, which will be more complicated,

$$
\frac{\partial \pi^{*}}{\partial \rho}=\frac{e^{r(t-T)} \lambda^{2} \sigma\left(-k+e^{(t-T)}(k+\lambda \rho \sigma)^{2}\right)}{x \gamma(k+\lambda \rho \sigma)^{2}} .
$$

If $t \in[0, T]$ is close enough to $T$, then

$$
\begin{gathered}
e^{(t-T)}(k+\lambda \rho \sigma)^{2}>k, \\
-k+e^{(t-T)}(k+\lambda \rho \sigma)^{2}>0,
\end{gathered}
$$

so that

$$
\frac{\partial \pi^{*}}{\partial \rho}>0
$$

which means the optimal investment strategy $\pi^{*}$ increasing with respect to the correlation coefficient $\rho$. However, as $t$ is close enough to 0 , especially for $t \in[0, T]$ small enough, then

$$
\begin{gathered}
e^{(t-T)}(k+\lambda \rho \sigma)^{2}<k, \\
-k+e^{(t-T)}(k+\lambda \rho \sigma)^{2}<0
\end{gathered}
$$

which shows that the optimal investment strategy $\pi^{*}$ decreases with respect to the correlation coefficient $\rho$, referring to Figure 4 which illustrates our conclusion.

Second, we analyze the efficient frontier in the distribution phase after retirement. Analysis of the efficient frontier in the accumulation phase before retirement is almost the same, so it is omitted. We fix the time $t$ and take appropriate value to $x$ and $l$ for the efficient frontier (49) which is a function of variance as the independent variable. Drawing a picture of the efficient frontier on the rectangular plane coordinate system of variance (Var) and expected return (Exp) for the different risk aversion levels $\gamma$, referring to Figure 5, they are convex curves and the expected return (Exp) smaller if the time $t$ takes larger.

Taking derivatives of variance (47) and by similar analysis as above we also have

$$
\frac{\partial \operatorname{Var}_{t, x, l}\left[X^{\pi^{*}}(T)\right]}{\partial \gamma}<0, \quad \frac{\partial \operatorname{Var}_{t, x, l}\left[X^{\pi^{*}}(T)\right]}{\partial t}<0 .
$$

The inequalities in (91) show that the variance decreases with respect to the risk aversion coefficient $\gamma$ and time $t$ when the fund size level and other parameters are fixed. Referring to Figure 6 shows the correctness of our conclusion. Variance as a measure of risk will be smaller if investor be more risk averse. Meanwhile, for more longer time investment, a risk aversion insurer will be more cautious, so the variance also decreases with the investment time $t$. 


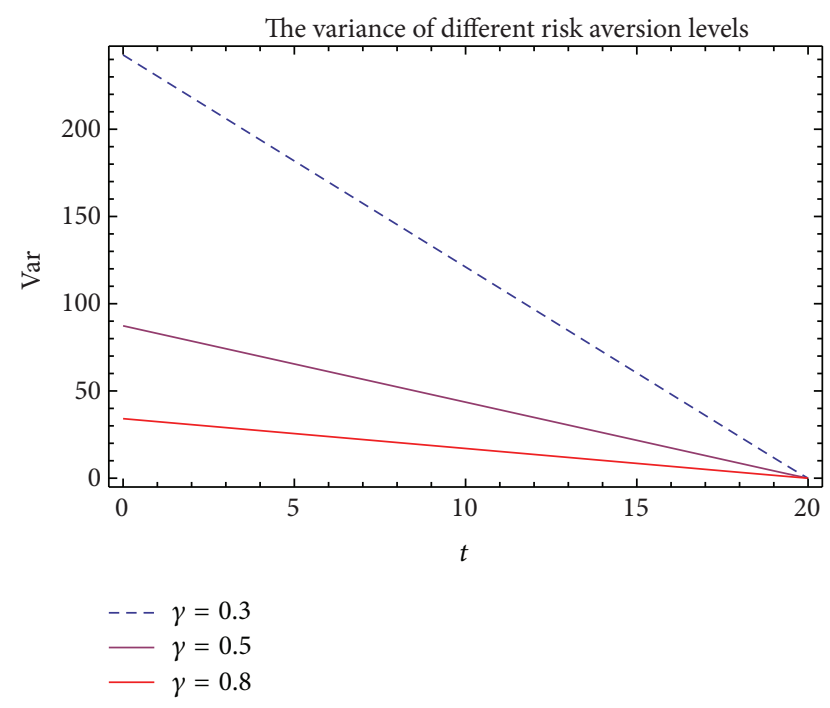

FIGURE 6: Variance with different risk aversion levels $\gamma$.

Through detailed comparison of the above figures, we also discover that different risk aversion levels can lead to surprising difference of the fund size variance (seeing Figure 6) and the more strict correlation of Brownian motions may result in fierce variation (seeing Figure 4) of the optimal investment strategy especially when time $t$ is close enough to $T$. So we should control risk aversion level and correlation coefficient of Brownian motions at an appropriate level for a DC pension plan.

\section{Conclusion}

The main innovation of this paper is merging defined contribution with annuity contract as a whole pension plan to study both accumulation phase before retirement and distribution phase after retirement in the mean-variance framework with the return of premiums clauses, which achieves the effect that both phases before and after retirement are "defined" to make the defined contribution plans even more portable and great convenience for insurance companies. The return of premiums clauses following the formulation of He and Liang [12], the time-consistent framework according to Li et al. [8] and inspired by the literature of Gao [1], we obtain the timeconsistent explicit solution by applying stochastic optimal control techniques under Heston's SV models. Numerical analysis illustrates our results and also deepens our knowledge or understanding of the research results.

\section{Conflict of Interests}

The authors declare that there is no conflict of interests regarding the publication of this paper.

\section{Acknowledgments}

This research was supported by the National Natural Science Foundation of China (Grant nos. 11201335 and 11301376) and the Humanity and Social Science Foundation of Ministry of Education of China (Grant no. 11YJC910007).

\section{References}

[1] J. Gao, "Optimal investment strategy for annuity contracts under the constant elasticity of variance (CEV) model," Insurance: Mathematics and Economics, vol. 45, no. 1, pp. 9-18, 2009.

[2] A. J. G. Cairns, D. Blake, and K. Dowd, "Stochastic lifestyling: optimal dynamic asset allocation for defined contribution pension plans," Journal of Economic Dynamics and Control, vol. 30, no. 5, pp. 843-877, 2006.

[3] N. Dokuchaev and X. Yu Zhou, "Optimal investment strategies with bounded risks, general utilities, and goal achieving," Journal of Mathematical Economics, vol. 35, no. 2, pp. 289-309, 2001.

[4] C. Blanchet-Scalliet, N. El Karoui, M. Jeanblanc, and L. Martellini, "Optimal investment decisions when time-horizon is uncertain," Journal of Mathematical Economics, vol. 44, no. 11, pp. 1100-1113, 2008.

[5] S. Faggian and F. Gozzi, "Optimal investment models with vintage capital: dynamic programming approach," Journal of Mathematical Economics, vol. 46, no. 4, pp. 416-437, 2010.

[6] D. Blake, D. Wright, and Y. Zhang, "Target-driven investing: optimal investment strategies in defined contribution pension plans under loss aversion," Journal of Economic Dynamics and Control, vol. 37, no. 1, pp. 195-209, 2013.

[7] H. Kraft, "Optimal portfolios and Heston's stochastic volatility model: an explicit solution for power utility," Quantitative Finance, vol. 5, no. 3, pp. 303-313, 2005.

[8] Z. Li, Y. Zeng, and Y. Lai, "Optimal time-consistent investment and reinsurance strategies for insurers under Heston's SV model," Insurance: Mathematics and Economics, vol. 51, no. 1, pp. 191-203, 2012.

[9] H. Markowitz, "Portfolio selection," Jornal of Finance, vol. 7, pp. 77-91, 1952.

[10] T. Bjork and A. Murgoci, "A general theory of Markovian time inconsistent stochastic control problems," Working Paper, Stockolm School of Economics, 2010.

[11] T. Bjork, A. Murgoci, and Z. Xunyu, "Mean-variance portfolio optimization with statedependent risk aversion," Mathematical Finance, vol. 24, no. 1, pp. 1-24, 2012.

[12] L. He and Z. Liang, "Optimal investment strategy for the DC plan with the return of premiums clauses in a mean-variance framework," Insurance: Mathematics and Economics, vol. 53, no. 3, pp. 643-649, 2013. 


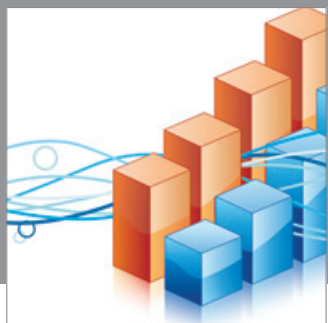

Advances in

Operations Research

mansans

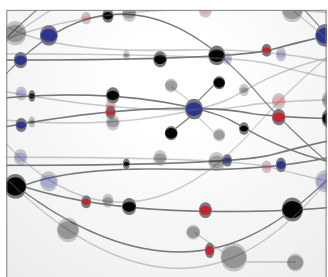

The Scientific World Journal
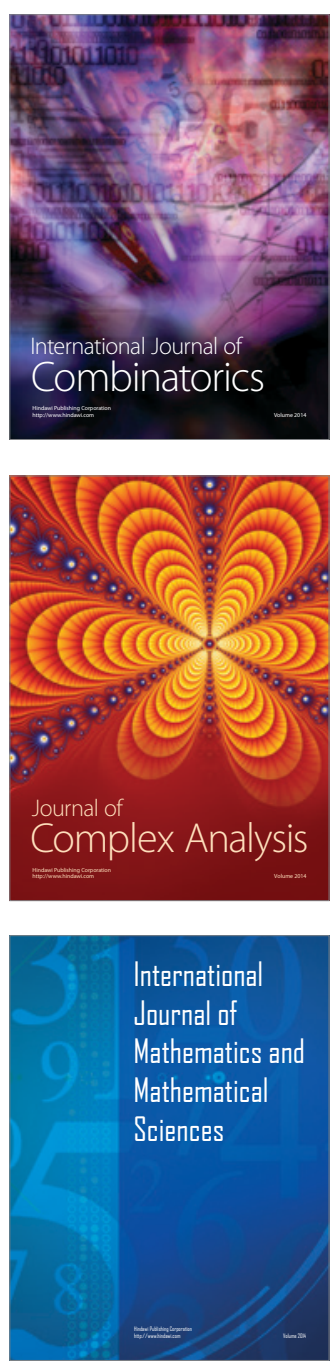
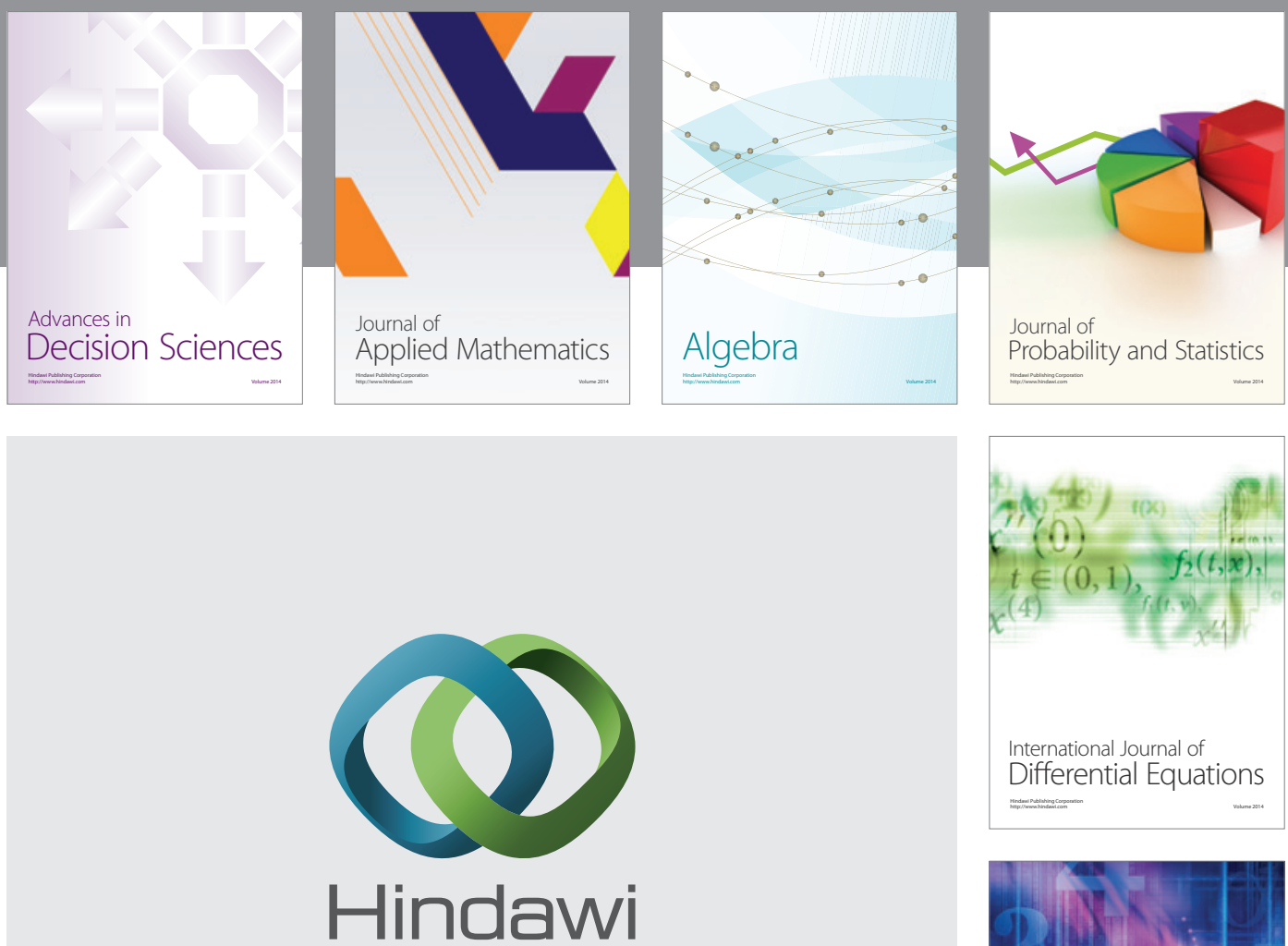

Submit your manuscripts at http://www.hindawi.com
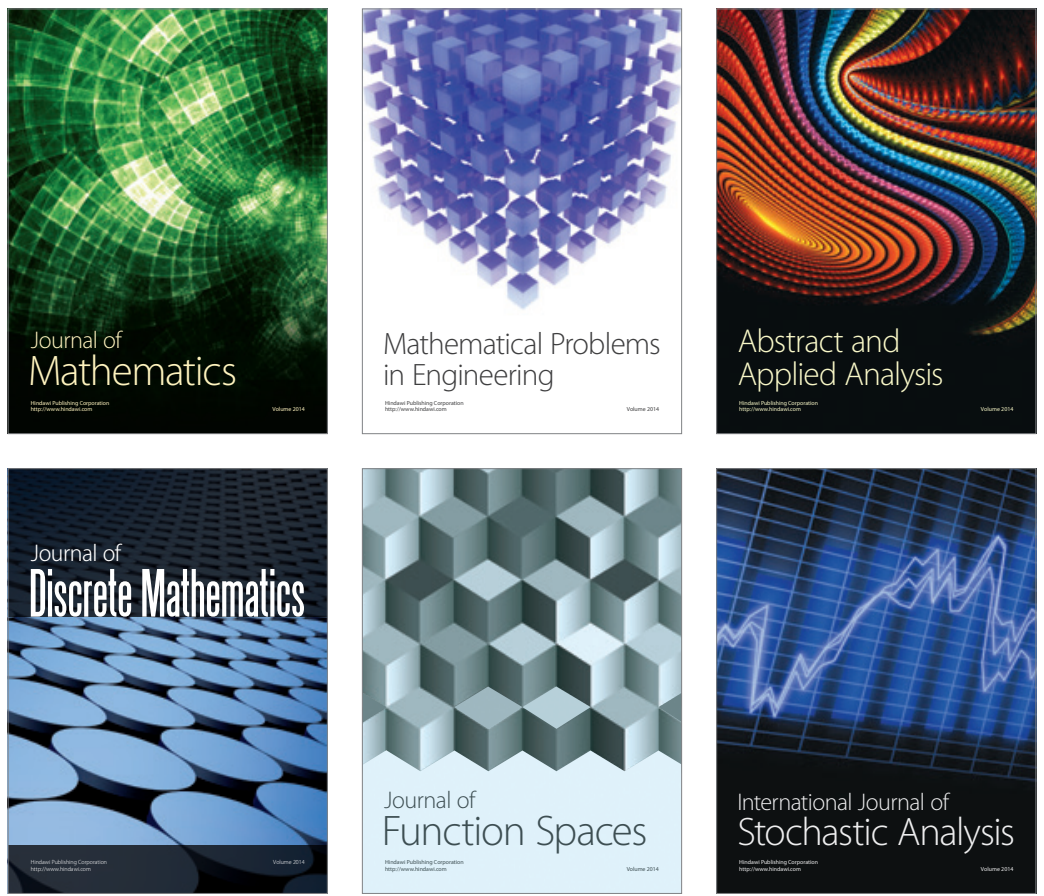

Journal of

Function Spaces

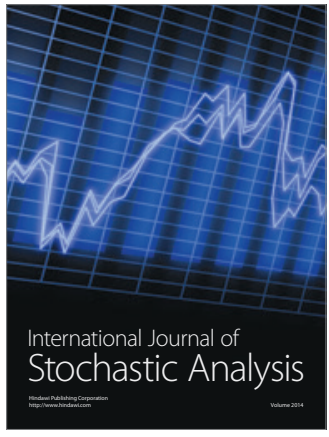

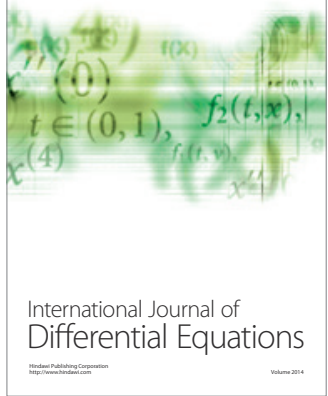
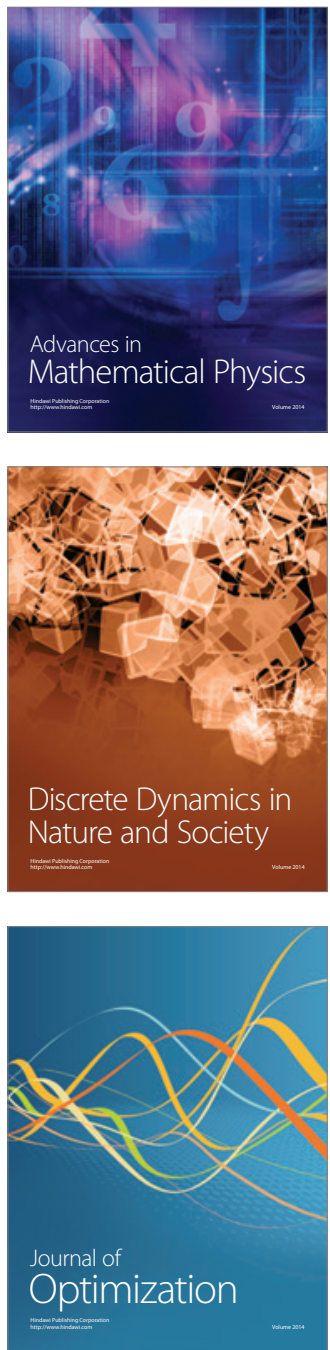